Rev, Saúde públ.. S. Paulo

$12: 1-15,1978$

\title{
MORTALIDADE POR CÂNCER NO ESTADO DE SÃO PAULO, BRASIL - 1970/1972
}

Edmur Flávio Pastorelo *

Sabina Léa Davidson Gotlieb *

\begin{tabular}{r|r|}
\hline RSPUB9/388 & \multicolumn{1}{|c|}{$\mid$} \\
\hline
\end{tabular}

Pastorelo, E. F. \& Gotlieb, S. L. D. Mortalidade por câncer no Estado de São Paulo, Brasil - 1970/1972, Rev. Saúde públ., S. Pallo, 12 : $1-15,1978$.

RESUMO: Apresenta-se a distribuição dos obitos por cancer, de acordo com as principats sedes primárias do tumor e Regióes Administrativas do Estado de São Paulo, em 1970/1972. Algumas Regiōes, apesar de não fugirem ao padrão homogêneo, relativamente à mortalidade por câncer no Estado, apresentam coexistência de tumores tipicos de áreas em nivel sócio-econômico baixo e em elevação. Isto poderia ser explicado por contarem estas Regióes com cidadespolo de desenvolvimento industrial.

Unitermos: Câncer, Sáo Paulo, Brasil. Mortalidade.

\section{NTRODU ÇĀO}

As estatisticas oficiais de mortalidade do Estado de São Paulo, por terem sido apresentadas segundo local de ocorrência do evento, faziam com que, na sua análise, surgissem conclusões distorcidas da situação da mortalidade em uma determinada área, devido aos fenômenos de invasão e evasão de óbitos.

A partir de 1970 , as tabulaçōes oficiais dos eventos vitais passaram a ser feitas por local de residência, sanando, assim, o problema acima referido e permitindo que o real risco de morte, pelas diferentes causas de óbito, viesse a ser conhecido, para os habitantes das áreas específicas do Estado.

Desse modo, o possível comportamento diferente observado no quadro da mortali- dade por causas, nessas áreas, levaria ao desencadeamento de raciocínio epidemiológico interpretativo que, à luz de informações posteriores, tentaria elucidar o mecanismo responsável pelas diferenças observadas.

Baseando-se nessas considerações e havendo particular interesse no estudo dos tumores malignos, procedeu-se à análise dessa causa de óbito no Estado de São Paulo.

O objetivo deste trabalho é identificar o padrão de mortalidade diferencial por câncer, de acordo com a sede primária da lesão, nas Regiōes Administrativas do Estado de São Paulo, no período de $1970 /$ 1972.

\footnotetext{
* Do Departamento de Epidemiologia da Faculdade de Saúde Pública da USP - Avenida Dr. Arnaldo, 715 - São Paulo, SP - Brasil.
} 
PAStorelo. E. F. \& GOTLIEB, S. L. D. M ortalidade por câncer no Estado de São Paulo, Brasil - 1970/1972. Rev. Saúde públ., S. Paulo. 12.1-15. 1978.

MATERIAL E MESTODOS

\section{Populagiono}

Utilizaram-se os daclos populacionais estimados por Pastorelo \& Gotliebo no cálculo dos coeficientes específicos de mortalidade por câncer segundo idade, sexo e sedes primárias da lesão, para os residentes das Regiōes Administrativas do Estado de São Paulo, no período 1970 a 1972.

Ao se analisar a composição percentual etária e por sexo das populaçóes das Regiōes Administrativas, notaram-se diferenças entre elas que trariam distorçōes na comparabilidade de seus riscos de morte por câncer. Utilizou-se, então, método da padronização direta desses coeficientes, tomando-se como população padrão, a do Estado de São Paulo, para o periodo em estudo.

\section{óbitos}

Foram utilizados os uados oficiais de óbitos do Estado de São Paulo de 1970, 1971 e 1972 coletados, compilados e codificados pelo Departamento de Estatistica da Secretaria de Economia e Planejamento do Estado de São Paulo. Esses dados encontram-se gravados em fitas magnéticas e a partir de cópias das mesmas, elaboraramse tabelas da distribuição dos óbitos segundo as variáveis: sexo, idade, local de residência e causas de morte baseadas na 8" Revisão da Classificação Internacional de Doenças e Causas de Morte ${ }^{5}$.

As causas apresentacias no decorrer deste trabalho são:

150 - Tumor malígno do esôfago

151 - Tumor malígno do estômago

153 - Tumor maligno do intestino grosso exceto reto

157 - Tumor malígno do pâncreas

161 - Tumor malígno da laringre
162 - Tumor maligno da traquéia, brônçuios e pulmão

174 - Tumor maligno da mama

180 - Tumor malígno do colo do útero

182.0 - Tumor malígno do corpo do biero

182.9 - Tumor maligno do útero sem especificar a parte

185 - Tunur maligno da próstata

195 - Tumor malígno de localizações mal definidas

196 - Tumor maligno secundário, ou não especificado dos gânglioss linfáticos

197 - Tumor malígno secundário dos aparelhos respiratório e digestivo

198 - Outros tumores malignos secundários

199 - Tumor maligno de localização não especificada

A apuração dos dados foi realizada no Centro de Computação Eletrônica da Universidade de São Paulo. Na programação foram utilizados a linguagem Fortran IV-G, o Sistema SPSS e o computador Burroughs-6700.

RESULTADOS E ANALISE

\section{Mortalidade proporcional - Principais} Causas de Morte.

Compreendendo $12.724,13.266$ e 13.674 óbitos de residentes no Estado de São Paulo. respectivamente, nos anos de 1970, $1971 \mathrm{e}$ 1972, os óbitos por tumores malígnos representaram a quinta causa de morte no sexo masculino e a quarta, no feminino, para o triênio analisado, excluindo-se o grupo de sintomas e estados morbidos mal definidos (Tabela 1). 
PASTORELO, E. F. \& GOTLIEB. S. L. D. M ortalidade por câncer no Estado de São Paulo. Brasil - 1970/1972. Rev. Satide puibl., S. P aulo, 12:1-15, 197 ?.

T A B E I A I

Mortalidade Proporcional (\%) segundo principais grupos de causa hásica de morte e sexo no Fistado de São Paulo. 1970/1972.

\begin{tabular}{|c|c|c|}
\hline $\mathrm{P}^{2}$ & Masculine & Feminino \\
\hline Infecciosas e Parasitárias (B1 a B18) & $13,28 \quad(2)$ & $12,82 \quad(2)$ \\
\hline Tumores malignos (B19) & $8.42 \quad(5)$ & $8,80 \quad(4)$ \\
\hline Cardiovasculares (B25 a B30) & 26,11 (1) & $28.65 \quad(1)$ \\
\hline Gripe, Pneumonias, Bronquite, Asma e Enfisema (B31 a B33) & $9,28 \quad(4)$ & $9,74 \quad(3)$ \\
\hline Perinatais (B43 a B44) & $6,62 \quad(6)$ & $6,79 \quad(5)$ \\
\hline Sintomas e estados mórbidos mal definidos (B45) & 10,13 & 10,78 \\
\hline Acidentes, Envenenamento e Violências (BE47 a BE50) & 10,11 & $3,95 \quad(6)$ \\
\hline Todas as outras & 16,05 & 17,47 \\
\hline
\end{tabular}

( ) Postos

* Classificação Internacional de Doenças e Causas de óbito - 8" Revisão - 1965.

Analisando-se o) comportamento da mortalidade proporcional por câncer nas $\mathrm{Re}$ giōes Administrativas do Estado (Tabela 2) observa-se que, excluindo o grupo B45. no sexo masculino, o câncer ocupa a terceira posição nas Regiões de Ribeirão Preto e Bauru. Nas Regiões de Campinas, São José do Rio Preto e Araçatuba está em quarto lugar. Para Grande São Paulo, Litoral, Sorocaba, Presidente Prudente e Marilia é a quinta causa de óbito, sendo a sexta, no Vale do Paraíba.

Para () sexo feminino (Tabela 3) é o câncer a terceira causa de óbito nas Regiōes de Campinas, Ribeirão Preto, Bauru, São José do Rio Preto e Araçatuba. Como quarta causa aparece nas Regióes da Grande São Paulo, Litoral, Sorocaba e Marilia, sendo nas Regiōes do Vale do Paraiba e Presidente Prudente a quinta causa de óbito.

Chama a atenção o fato de que o grupo dos sintomas e estados mórbidos mal definidos apresenta-se em torno de $10 \%$ do total de óbitos no Estado de São Paulo, para o periodo (Tabela 1 ).
Chega a altos valores, como por exempio na Região de Sorocaba, onde $22,1 \%$ dos óbitos masculinos e $24,9 \%$ dos femininos são classificados segundo esse grupo de causas de morte (Tabelas 2 e 3), refletindo mal preenchimento do atestado de óbito e possível mal diagnóstico, incluindo também os óbitos sem assistência médica. Isto leva a indagações a respeito da acurácia da causa básica contida no atestado de óbito, fonte das informações oficiais.

Para o Distrito de São Paulo, através de pesquisas levadas a efeito, como a realizada por Puffer e Griffith ${ }^{7}$, subsidios foram fornecidos para as devidas correçōes. Assim, um terço dós óbitos assinalados como sintomas e estados mórbidos mal definidos (B45), após correção da causa básica contida no atestado de óbito, receberam classificação mais precisa. Como, para os dados oficiais de óbito do Estado de São Paulo, não há elementos que avaliem a magnitude dessa imprecisão, as informações apresentadas neste trabalho deverão ser apreciadas tendo-se em mente as deficiências acima referidas. 
PASTORELO, E. F. \& GOTLIEB, S. L. D. Mortalidade por câncer no Estado de São Paulo, Brasil - 1970/1972. Rev. Saúde públ., S. Paulo, 12:1-15, 1978.

TA BELA 2

Mortalidade proporcional (\%) no sexo masculino, segundo principais causas de óbito e Regióes Administrativas do Estado de São Paulo, 1970/1972.

\begin{tabular}{|c|c|c|c|c|c|c|c|c|}
\hline Causas* & B1-B18 & B19 & B25-B30 & B:31-B33 & $\mathrm{B} 43-\mathrm{B} 44$ & B45 & $\mathrm{BE} 47-\mathrm{BE} 50$ & outras \\
\hline Grande São Paulo & 14,3 & 8,9 & 25,1 & 11,3 & 6,8 & 5,4 & 11,4 & 16.8 \\
\hline Litoral & 11,9 & 8,9 & 25,5 & 9,5 & 5,8 & 9,1 & 11,9 & 17,4 \\
\hline Vale do Paraiba & 13,2 & 6.1 & 22,9 & 7,1 & 7,7 & 17,5 & 9,9 & 15,6 \\
\hline Sorocaba & 11,3 & 6,2 & 24,1 & 9,5 & 5,5 & 22,1 & 7,5 & 13,8 \\
\hline Campinas & 11.1 & 9.2 & 31,0 & 7,6 & 6,2 & 8,5 & 9,4 & 17,0 \\
\hline Ribeirão Preto & 13,2 & 9.2 & 31,2 & 6,1 & 6,7 & 9,6 & 8,7 & 5,3 \\
\hline Bauru & 11,5 & 9,3 & 29,8 & 6,8 & 7,1 & 10,8 & 7,6 & 17,1 \\
\hline S. José do Rio Preto & 12,3 & 8,1 & 25,0 & 6,1 & 6,4 & 20,3 & 8,2 & 13,6 \\
\hline Araçatuba & 13,9 & 7.9 & 22,7 & 7,0 & 7,2 & 19,7 & 9,3 & 12,3 \\
\hline Pres. Prudente & 13,8 & 6,9 & 21,1 & 7,3 & 6,9 & 20,5 & 8,7 & 14,8 \\
\hline Marf́lia & 15,0 & 6,7 & 26.0 & 7,3 & 6,5 & 16,7 & 7,5 & 14,3 \\
\hline
\end{tabular}

* Classificação Internacional de Doenças e Causas de óbito - $8^{\mathrm{n}}$ Revisão $-1965^{5}$

T A B E L A 3

Mortalidade proporcional (\%) no sexo feminino, segundo principais causas de óbito e Regióes Administrativas do Estado de São Paulo - 1970/1972.

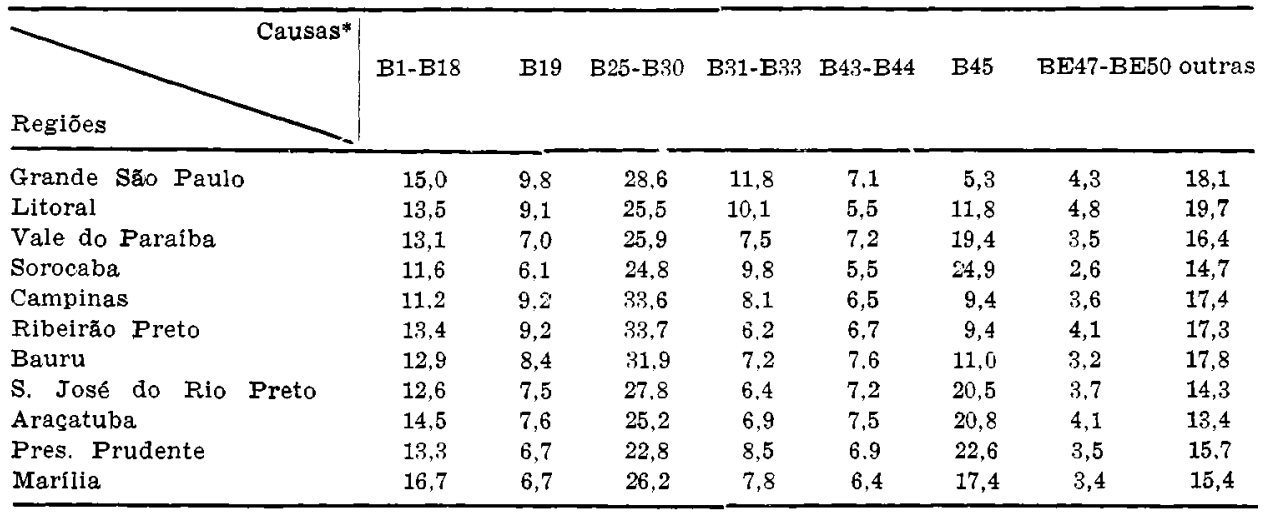

* Classificação Internacional de Doenças e Causas de óbito $-8^{*}$ Revisão -1965.

\section{Mortalidade Proporcional - Sedes primárias da lesão}

Observando-se a distribuição percentual dos óbitos femininos de câncer segundo as sedes primárias da lesão, para as Regiōes Administrativas (Tabela 4), nota-se que $n$ câncer da mama é a principal causa de óbito na Região da Grande São Paulo $15,7 \%$. Para as Regióes do Litoral e Bauru esta posição é ocupada pelo câncer do útero como um todo, com os seguintes valores: $15,4 \%$ e $17,2 \%$, respectivamente. Nas demais Regiões, é o câncer do estômago que ocupa o primeiro lugar, com valores variando de $19,8 \%$ para Marília a $16.5 \%$ no Vale do Paraiba. Além dessas citadas sedes primárias da lesão, encontram-se também entre as principais: intestino gros- 
PASTORELO, E. F. \& GOTLIEB, S. L. D. Mortalidade por câncer no Estado de São Paulo, Brasil - 1970/1972. Rev. Saúde puibl., S. Paulo, $12: 1-15,1978$.

so exceto reto, traquéia; brônquios e pulmão, pâncreas e esôfago.

No sexo masculino, a distribuição percentual dos óbitos de câncer segundo as sedes primárias da lesão (Tabela 5), apresenta o câncer do estômago como principal causa de óbito em todas as Regióes Administra- tivas, apresentando valores entre $33,8 \%$ a $20,3 \%$, respectivamente, para as Regiões de Marilia e Litoral. Sucessivamente aparecem traquéia, brônquios e pulmão e esôfago nos segundo e terceiro postos, para todas as Regiōes, exceto a de Presidente Prudente, onde ocorre inversão. As demais

T A B E L A 4

Mortalidade proporcional (\%) no grupo câncer (140-209) para o sexo feminino, segundo principais sedes primárias da lesão e Regiões Administrativas do Estado de São Paulo. $1970 / 1972$.

\begin{tabular}{|c|c|c|c|c|c|c|c|c|c|}
\hline Regiōes & Causas* & $\begin{array}{c}\text { Esôfago } \\
(150)\end{array}$ & $\begin{array}{l}\text { Esto- } \\
\text { mago } \\
(151)\end{array}$ & $\begin{array}{c}\text { Intest. } \\
\text { Grosso } \\
(153)\end{array}$ & $\begin{array}{c}\text { Pâncreas } \\
\text { (157) }\end{array}$ & $\begin{array}{l}\text { Pulmão } \\
\text { (162) }\end{array}$ & $\begin{array}{l}\text { Mama } \\
\text { (174) }\end{array}$ & $\begin{array}{l}\text { Uttero T } \\
\text { como to- } \\
\text { do }(180 . \\
182.0 \text { e } \\
182.9)\end{array}$ & $\begin{array}{l}\text { Todas as } \\
\text { outras } \\
\end{array}$ \\
\hline Grande São Paulo & & 1,5 & 14,5 & 6,1 & 3,1 & 4,7 & 15,7 & 13,4 & 41,0 \\
\hline Litoral & & 1,4 & 14,5 & 7,5 & 2,7 & 3,9 & 15,3 & 15,4 & 39,8 \\
\hline Vale do Paraíba & & 3,2 & 16,5 & 8.2 & 1,7 & 4,4 & 10,9 & 13,7 & 41,4 \\
\hline Sorocaba & & 1,6 & 17.1 & 6,4 & 2,6 & 4,8 & 11,9 & 16,5 & 39,1 \\
\hline Campinas & & 1,8 & 17,1 & 6,6 & 3,1 & 4,7 & 12,6 & 12,0 & 42,1 \\
\hline Ribeirão Preto & & 1.3 & 17.2 & 6,0 & 2,7 & 4,5 & 13,8 & 13,1 & 41,4 \\
\hline Bauru & & 3,3 & 14,2 & 5,4 & 3,8 & 6,3 & 10,7 & 17,2 & 39,1 \\
\hline S. José Rio Preto & & 1,5 & 17,3 & 7,9 & 2,7 & 4,0 & 13,1 & 11,4 & 42,1 \\
\hline Araçatuba & & 3.2 & 19,2 & 7,8 & 3,2 & 5,2 & 8,7 & 16,4 & 36,3 \\
\hline Pres, Prudente & & 3,1 & 16,8 & 8,2 & 1,8 & 3,4 & 10,3 & 13,6 & 42,8 \\
\hline Marília & & 3,2 & 19,8 & 4,8 & 4,4 & 4,4 & 9,8 & 14,6 & 39,0 \\
\hline
\end{tabular}

* Classificação Internacional de Doenças e Causas de óbito - 8* Revisão - 1965.

TABELA 5

Mortalitade proporcional $(\%)$ no grupo câncer (140-209) para o sexo masculino, segundo principais sedes primárias da lesão e Regiōes Administrativas do Estado de São Paulo, $1970 / 1972$.

\begin{tabular}{|c|c|c|c|c|c|c|c|c|}
\hline 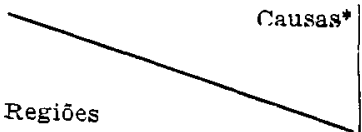 & $\begin{array}{c}\text { Esôfago } \\
(150)\end{array}$ & $\begin{array}{l}\text { Estô- } \\
\text { mago } \\
(151)\end{array}$ & $\begin{array}{c}\text { Intest. } \\
\text { Grosso } \\
\text { (153) }\end{array}$ & $\begin{array}{l}\text { Pâncreas } \\
\text { ((157) }\end{array}$ & $\begin{array}{c}\text { Laringe } \\
\text { (161) }\end{array}$ & $\begin{array}{c}\text { Pulmão } \\
\text { (162) }\end{array}$ & $\begin{array}{c}\text { Próstata } \\
\text { (185) }\end{array}$ & $\begin{array}{r}\text { Todas } \\
\text { as outras }\end{array}$ \\
\hline Grande São Paulo & 6,8 & 23,3 & 4,0 & 3,3 & $\mathbf{5 , 0}$ & 13,0 & 5,8 & 38,8 \\
\hline Litoral & 7,8 & 20,3 & 4,5 & 2,6 & 6,3 & 11,0 & 5,3 & 42,2 \\
\hline Vale do Paraíba & 7,2 & 31,6 & 4,4 & 3,0 & 3,4 & 9,4 & 3,6 & 37,4 \\
\hline Sorocaba & 7,2 & 29,4 & 3,2 & 2,0 & 4,1 & 8.5 & 4,6 & 37,4 \\
\hline Campinas & 6,4 & 26,1 & 3,2 & 3,1 & 5,5 & 11,1 & 5,7 & 38,9 \\
\hline Ribeirão Preto & 6,5 & 27,8 & 4,1 & 3,1 & 4,3 & 9,8 & 6,3 & 38,1 \\
\hline Bauru & 8,2 & 27,1 & 4.7 & 4,0 & 3,1 & 9.7 & 6,9 & 36,3 \\
\hline S. José Rio Preto & 8,1 & 24,1 & 4,7 & 3,7 & 4,2 & 9,1 & 5,4 & 40,7 \\
\hline Araçatuba & 7,2 & 30,2 & 5,4 & 3,8 & 3,2 & 8,0 & 4,6 & 37,6 \\
\hline Pres. Prudente & 8,8 & 32,3 & 3,3 & 2,4 & 3,7 & 7,5 & 5,9 & 36,1 \\
\hline Marília & 6,5 & 33,8 & 2,8 & 3,3 & 3,1 & 8,4 & 3.9 & 38,2 \\
\hline
\end{tabular}

* Classificação Internacional de Doenças e Causas de óbito - 8" Revisão - 1965. 
PASTORELO, E. F. \& GOTLIEB, S. L. D. Mortalidade por câncer no Estado de São Paulo, Brasil - 1970/1972. Rev. Saude pribl., S. P nulo, 12:1-15, 1978.

sedes primárias da lesão são por ordem de importância: próstata, laringe, intestino grosso exceto reto e pâncreas.

Nota-se a elevada proporção com que aparecem no grupo câncer (140-209) os tumores de localizações mal definidas (195), tumor malígno secundário ou não especificado dos gânglios linfáticos (196), tumor malígno secundário dos aparelhos respiratório e digestivo (197), outros tumores malígnos secundários (198) e tumor maligno de localização năo especificada (199). Os valores apresentam-se entre $10,5 \%$ e $7,8 \%$, respectivamente, nas Regiões de Sorocaba e Araçatuba, no sexo masculino e $13,6 \%$ na Região de Campinas e $9,1 \%$ na de Araçatuba, no sexo feminino. (Tabela 6).

TA B E L A 6

Mortalidade proporcional (\%) no grupo câncer (140-209) segundo sexo, categorias selecionadas e Regiões Administrativas do Estado de São Paulo, 1970/1972.

\begin{tabular}{|c|c|c|c|c|c|c|c|}
\hline \multirow[b]{2}{*}{ Regiōes } & \multirow{2}{*}{$\frac{\text { Sexn }}{\text { Causas* }}$} & \multicolumn{3}{|c|}{ Masculino } & \multicolumn{3}{|c|}{ Feminino } \\
\hline & & $\begin{array}{c}196-197 \\
198\end{array}$ & $195-199$ & TOTAL & $\begin{array}{c}196-197 \\
198\end{array}$ & $195-199$ & TOTAL \\
\hline Grande São Paulo & & 4.0 & 4.8 & 8.8 & 5.2 & 6,0 & 11.2 \\
\hline Litoral & & 6,1 & 3.8 & 9.9 & $\mathbf{5 , 6}$ & 5.7 & 11,3 \\
\hline Vale do Parafba & & 5,6 & 4,7 & 10,3 & 7,9 & 4,5 & 12,4 \\
\hline Sorocaba & & 5,4 & 5,1 & 10,5 & 8.1 & 4,8 & 12,9 \\
\hline Campinas & & 5,8 & 4,2 & 10,0 & 8,2 & 5,4 & 13,6 \\
\hline Ribeirão Preto & & 4,4 & 3.5 & 7.9 & 6.9 & 4,0 & 10,9 \\
\hline Bauru & & 5,2 & 2.8 & 8.0 & 10.6 & 1,7 & 12.3 \\
\hline S. José Rio Preto & & 5.8 & 3.5 & 9.3 & 7,6 & 3,4 & 11,0 \\
\hline A raçatuba & & 4,6 & 3,2 & 7,8 & 5,6 & 3.5 & 9,1 \\
\hline Pres. Prudente & & 5,4 & 2,9 & 8,3 & 6,0 & 4,1 & 10.1 \\
\hline Marília & & 5,1 & 4,4 & 9.5 & 8.2 & 3,8 & 12.0 \\
\hline
\end{tabular}

* Classificação Intemacional de Doenças "Causas de obito - 8n Revisão - 1965.

Tal fato representa existencia de falhas globais tanto no diagnóstico como no preenchimento do atestado de óbito, relativamente à causa básica de morte, vindo subestimar a real magnitude das sedes primárias e ou bem definidas dos diversos tumores.

Semelhante apreciação pode ser feita em relação aos tumores do útero como um todo $(180,182.0$ e 182.9). A categoria tumor malígno do útero sem especificar a parte apresenta-se com valores bem elevados chegando mesmo a ultrapassar, em todas as Regiões, os valores relativos aos tumores do corpo do útero e, em $80 \%$ delas, ultrapassam a frequiência com que aparecem os tumores do colo uterino (Tabela 7).
Neste sentido, Puffer e Griffith : mostraram que, após correções efetuadas na causa básica de óbito fornecida pelos atestados oficiais de 1962-64, do Distrito de São Paulo, 85\% dos óbitos atestados como câncer do útero sem especificar a parte, na realidade, eram neoplasias malignas do colo uterino.

Schoenberg e Bailars utilizancio a mesma metodologia, para os óbitos dos Estados Unidos em 1966, afirmaram que, após as devidas correções, o coeficiente de mortalidade por câncer do colo uterino passou de 7,7 para 8,3 por cem mil habitantes. Concluiram ainda, que o percentual dos casos corrigidos em favor de câncer do colo uterino se fez sentir mais nos grupos 
PASTORELO, E. F. \& GOTLIEB, S. L. D. Mortalidade por câncer no Estado de São Paulo. Brasil - 1970/1972. Rev, Saúde públ., S. Paulo, 12:1-15, 1978.

etários jovens, decrescendo com o avançar das idades, porém não especificaram o ganho em fidedignidade do diagnóstico para cada grupo etário. $\mathrm{Na}$ impossibilidade de aplicar essas informações na correção da alta freqüencia com que aparecem os tumores do útero sem especificar a parte (182.9), no Estado de São Paulo, optou-se por apresentar os dados como tumores do útero como um todo, a fim de que não ocorra uma falsa apreciação dos resultados aqui apresentados.

T A B E L A

Mortalidade proporcional (\%) no grupo câncer (140-209), para o sexo feninino segundo alyumas sedes primárias do aparelho genital e Regiões Administrativas do Estado de São Paulo. 1970/1972:

\begin{tabular}{|c|c|c|c|}
\hline Regiōes & $\begin{array}{l}\text { colo uterino } \\
\text { (180) }\end{array}$ & $\begin{array}{c}\text { corpo do útero } \\
(182.0)\end{array}$ & $\begin{array}{c}\text { útero, sem especificar } \\
\text { a parte. } \\
(182.9)\end{array}$ \\
\hline Grande Săo Paulo & 5,5 & 0,8 & 7,1 \\
\hline Litoral & 8,0 & 0,6 & 6,8 \\
\hline Vale do Paraíba & 5,4 & 0,6 & 7,7 \\
\hline Sorocaba & 7,1 & 0,4 & 9,0 \\
\hline Campinas & 5,1 & 0,5 & 6.4 \\
\hline Ribeirão Preto & 6,5 & 1.3 & 5,3 \\
\hline Bauru & 4,4 & 1.9 & 10,9 \\
\hline S. José do Rio Preto & 5,4 & $0 . \because$ & 5.7 \\
\hline Araçatuba & 4.4 & 1,2 & 10.8 \\
\hline Presidente Prudente & 6,2 & 1,0 & 6,4 \\
\hline Marflia & 6,0 & 0,8 & 7.8 \\
\hline
\end{tabular}

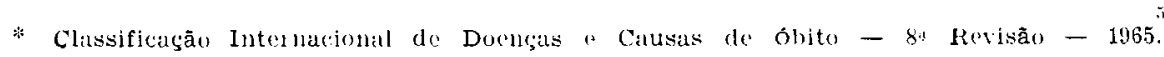

A tabela 8 mostra a ordenaçào das principais sedes primárias dos tumores malignos no Estado de São Paulo. No sexo masculino, $25 \%$ dentre os óbitus por câncer foi devido a tumor malígno do estômago, seguindo-se o pulmão com $11,4 \%$. Câncer de estômago é também a principal sede primária, no sexo feminino, com $15,5 \%$ dos óbitos, sendo mama, com $14,1 \%$, a segunda.

Mortalidade diferencial entre as $R e-$ gioes Administrativas: coeficientes de morlidade padronizados.

Para uma correta comparabilidade entre os riscos de morte por tumores malígnos, segundo suas sedes primárias mais freqüentes, entre as várias Regiōes Administrativas do Estado de São Paulo, utilizaram-se os coeficientes de mortalidade padronizados, que estão apresentados, para o sexo mas- culino, na Tabela 9 e para o teminino, na Tabela 10 .

Para o sexo masculino, $u$ cáncer de estômago aparece indiscutivelmente conı o grande risco de morte para todas as Regiōes, apresentando valores próximos a 21 por cem mil habitantes, na maioria delas, sendo que, apenas na Região de São José do Rio Preto apresenta valor igual a 15,40 por cem mil habitantes. Segue-se o câncer do pulmão com grande variabilidade do seu risco de morte nas várias Regiōes, com valores extremos de 11,41 por cem mil habitantes na Grande São Paulo a 4,35 por cem mil habitantes em Presidente Prudente. Tumor malígno do esôfago a seguir, com coeficiente de mortalidade de 6,89 por cen mil habitantes no Litoral e como Região de menor risco, Marilia com coeficiente de 4,19 por cem mil habitantes. 
PASTORELO, E. F. \& GOTLIEB, S. L. D. Mortalidade por câncer no Estado de São Paulo, Brasil - 1970/1972. Rev. Saude puibl., S. Paulo, 12:1-15, 1978.

TA B E L A 8

Mortalidade proporcional (\%) no grupo câncer (140-209) segundo sexo e algumas sedes primárias da lesão, no Estado de São Paulo, 1970/1972.

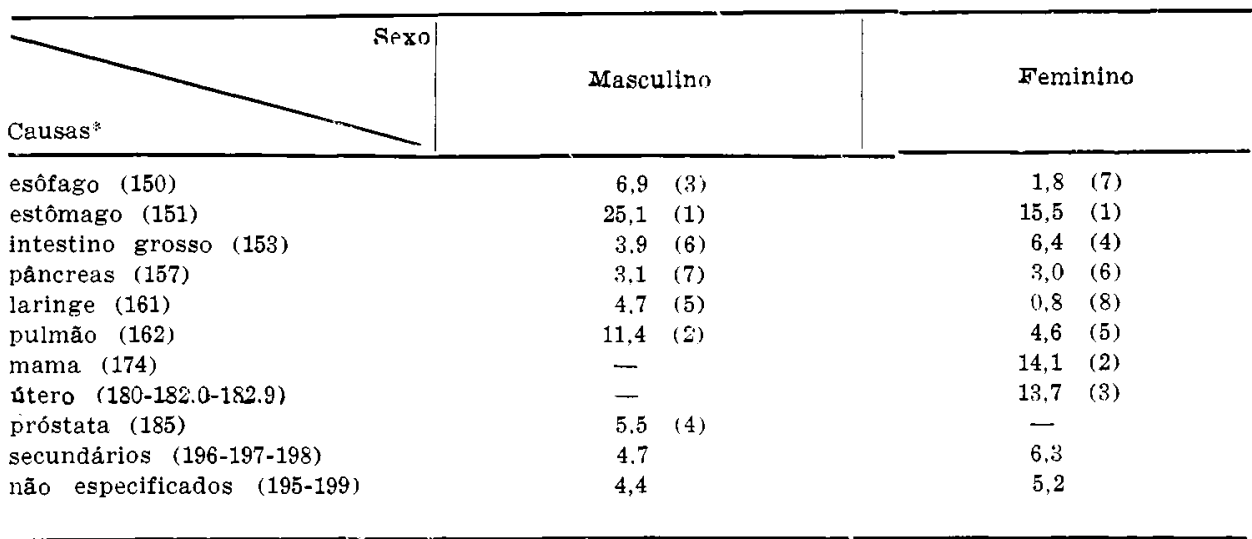

( ) - Postos

* Classificação Internacional de Doenças e Causas de óbito $-8^{4}$ Revisão $-1965^{5}$

T A B E L A 9

Coeficientes padronizados* de mortalidade por câncer (140-209) segundo algumas sełes primárias da lesão, para a população masculina das Regiões Administrativas do Estado de São Paulo, 1970/1972 (X 100.000 habitantes).

\begin{tabular}{|c|c|c|c|c|c|c|c|}
\hline Regiōes & $\begin{array}{l}\text { Esô- } \\
\text { fago } \\
(150)\end{array}$ & $\begin{array}{l}\text { Estô- } \\
\text { mago } \\
(151)\end{array}$ & $\begin{array}{l}\text { Intest. } \\
\text { grosso } \\
\text { (153) }\end{array}$ & $\begin{array}{l}\text { Pâncreas } \\
\text { (157) }\end{array}$ & $\begin{array}{l}\text { Laringe } \\
\text { (161) }\end{array}$ & $\begin{array}{l}\text { Pulmão } \\
\text { (162) }\end{array}$ & $\begin{array}{l}\text { Próstata } \\
\text { (185) }\end{array}$ \\
\hline Grande S. Paulo & 5,90 & 20,42 & 3,49 & 2,88 & 4,28 & 11,41 & 5,25 \\
\hline Litoral & 6,89 & 18,01 & 3,98 & 2,27 & 5,55 & 9,77 & 4,74 \\
\hline Vale do Paraíba & 4,83 & 21,31 & 2,93 & 1,97 & 2,24 & 6,31 & 2,36 \\
\hline Sorocaba & 4,56 & 18,48 & 1,99 & 1,30 & 2,58 & 5,28 & 2,74 \\
\hline Campinas & 5,07 & 20,45 & 2,50 & 2,43 & 4,42 & 8,73 & 4,34 \\
\hline Ribeirão Preto & 4,92 & 21,01 & 3,10 & 2,33 & 3,23 & 7,43 & 4,58 \\
\hline $\begin{array}{l}\text { Bauru } \\
\text { S. José do Rio }\end{array}$ & 6,30 & 20,85 & 3,74 & 3,07 & 2,46 & 7,76 & 4,94 \\
\hline Preto & 5,26 & 15,40 & 2,95 & 2,35 & 2,74 & 5,80 & 3,33 \\
\hline Araçatuba & 4,71 & 20,13 & 3,59 & 2,55 & 2,15 & 8,36 & 3,05 \\
\hline Pres. Prudente & 4,97 & 18,67 & 1,88 & 1,35 & 2,12 & 4,35 & 3,40 \\
\hline Marilia & 4,19 & 21,56 & 1,83 & 2,08 & 2,02 & 5,43 & 2,37 \\
\hline Est. São Paulo & 5,49 & 19,96 & 3,08 & 2,48 & 3,73 & 8.95 & 4.39 \\
\hline
\end{tabular}

Relativamente ao câncer da próstata é na Região da Grande São Paulo que se encontra o maior risco de morte, 5,25 por cem mil habitantes, sendo por sua vez, o menor coeficiente de mortalidade 2,35 por cem mil habitantes, no Vale do Paraiba. Quanto à ordenação, pela magnitude dos riscos de morte por essas causas já men- 
PASTORELO, E. F. \& GOTLIEB, S. L. D. Mortalidade por câncer no Estado de São Paulo, Brasil - 1970/1972. Rev. Saúde públ., S. Paulo, 12:1-15, 1978.

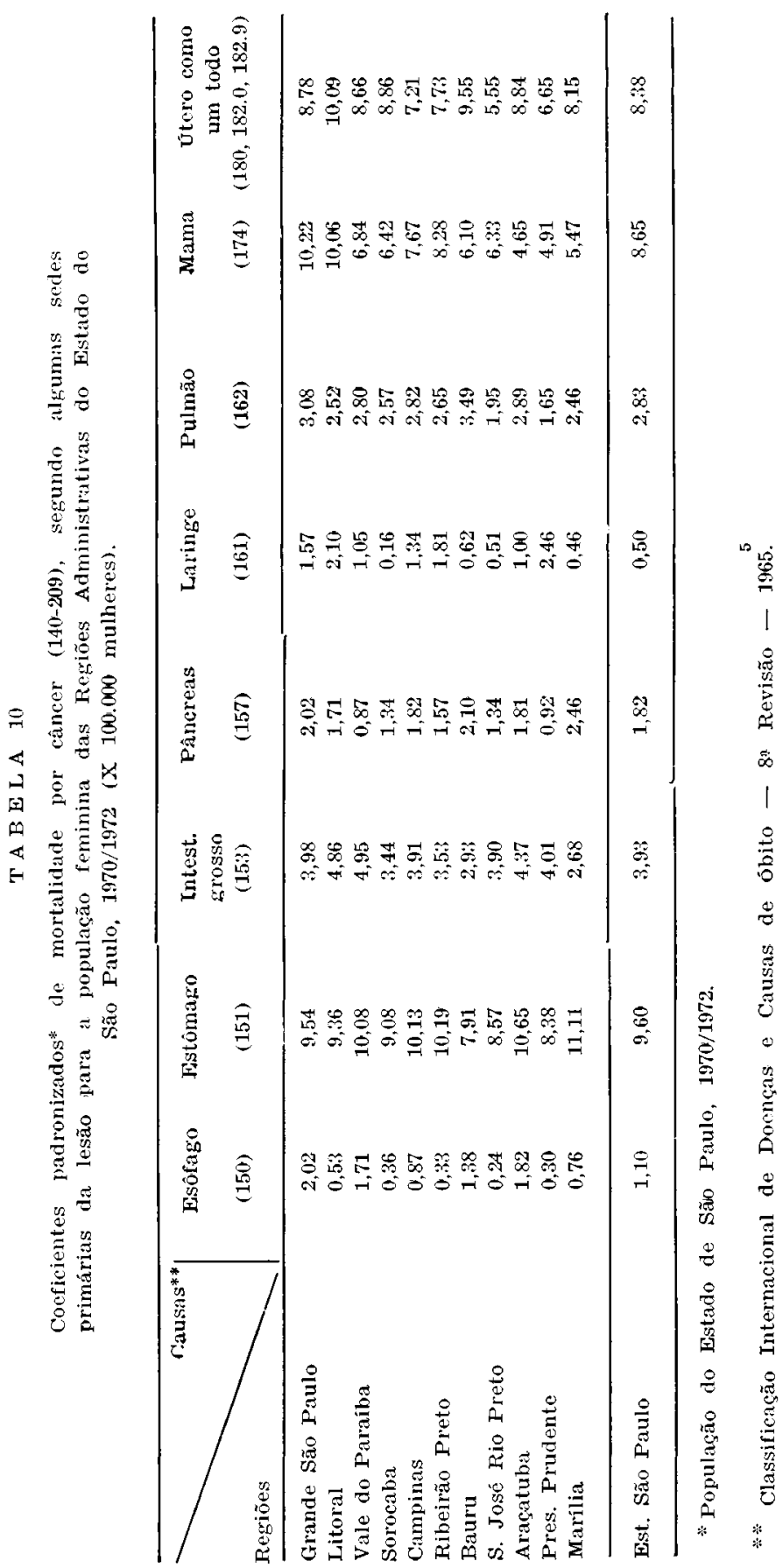


PASTORELO, E. F. \& GOTLIEB, S. L. D. Mortalidade por câncer no Estado de São Paulo, Brasil - 1970/1972. Rev. Saúde públ., S. P aulo, 12:1-15, 1978.

cionadas, há quase uma homogeneidade entre as Regiōes, porém, o mesmo não ocorrendo para o câncer da laringe, do intestino grosso exceto reto e do pâncreas que apresentam uma acentuada discordância classificatória.

Para o Estado, a ordenação dessas causas de morte, como se esperava, segue os padrões apresentados nas Regióes Administrativas. Assim, é o câncer do estômago o principal risco de morte dentre os tumores malígnos, com coeficiente de mortalidade de 19,96 por cem mil habitantes.

Seguem-se tumores malignos da traquéia, brônquios e pulmāo e do esôfago com coeficientes de mortalidade de 8,95 e 5,49 por cem mil habitantes, respectivamente. Por ordem de importância, as demais sedes primárias da lesão são: próstata, laringe, intestino grosso exceto reto e pâncreas.

No sexo feminino, o risco de morte segundo as sedes primárias da lesāo não apresenta, entre as Regiōes Administrativas, a homogeneidade de ordenação, como a ocorrida no sexo masculino. Assim, existe certa concordância em relação ao câncer do intestino grosso exceto reto, do pulmão e dò pâncreas, que ocupam respectivamente a quarta, quinta e sexta posição na maioria das Regiōes Administrativas. Com respeito às principais sedes primárias: câncer de mama, do estômago e do útero como um todo existe uma variabilidade como principal risco de morte. Câncèr de mama, primeira causa dentre os óbitos por câncer na Grande São Paulo, apresenta-se como maior risco de morte dentre as Regiões, $(10,22$ por cem mil mulheres), sendo que, Araçatuba é a Região que apresenta o menor risco por essa causa, da ordem de 4,65 por cem mil mulheres. Por sua vez, a Região de Bauru apresenta como a primeira causa de óbito, dentre os tumores malignos, útero como um todo, com coeficiente de mortalidade de 9,55 por cem mil mulheres. A Região de São José do Rio Preto, por seu lado, apresenta o menor risco de morte por essa causa, com coeficiente igual a 5,55 por cem mil mulheres.
Chama a atenção, a Região do Litoral que apresenta valores dos coeficientes de mortalidade por câncer de mama e do útero como um todo, elevados e praticamente iguais, en torno de 10,1 por cem mil mulheres.

Todas as outras Regiōes apresentanı o câncer do estômago como principal risco, variando entre 11,11 por cem mil mulheres em Marilia e 8,38 por cem mil mulheres em Presidente Prudente, sendo que o primeiro é o maior coeficiente dentre as Regiões. O menor coeficiente para essa causa é o de Bauru com valor de 7,91 por cem mil mutheres.

Relativamente ao Estado como um todo, os principais riscos de morte segundo sedes primárias selecionadas dentre os tumores malígnos são devidos a: neoplasia do estômago - 9,60 por cem mil mulheres, da mama $-8,65$ por cem mil mulheres e do útero como um todo $-8,38$ por cen mil mulheres.

\section{Comparaçăo entre riscos mascullılo $e$ feminino}

Uma vez conhecida a predominância de determinadas sedes primárias dos tumores malignos, em função do sexo, analisou-se esse comportamento nas Regióes Administrativas do Estado de São Paulo.

Assim, na Tabela 11, depreende-se () fato de que o câncer do estômago se apresenta em todas as Regiões Administrativas. com risco duas vezes maior para o sexo masculino. Por sua vez, o câncer do intestino grosso exceto reto é de tendência mais acentuada no sexo feminino, apresentando valores, para a razão homens/mulheres, entre 0,5 e 0,9 , com única exceção na Região de Bauru, onde o risco no sexo masculino é 1,3 vezes maior.

O tumor maligno do pulmão predonina no sexo masculino, porém, com distribuição regional marcada por uma variação mais ampla do que a apresentada pelo câncer do estômago. Desse modo, tem-se na Região do Litoral um risco praticamente qua- 
PAStorelo, E. F. \& GOTLieb. S. L. D. Mortalidade por câncer no Estauo de São Paulo, Brasil - 1970/1972. Rer. Saúde públ., S. Paulo. 12:1-15, 1978.

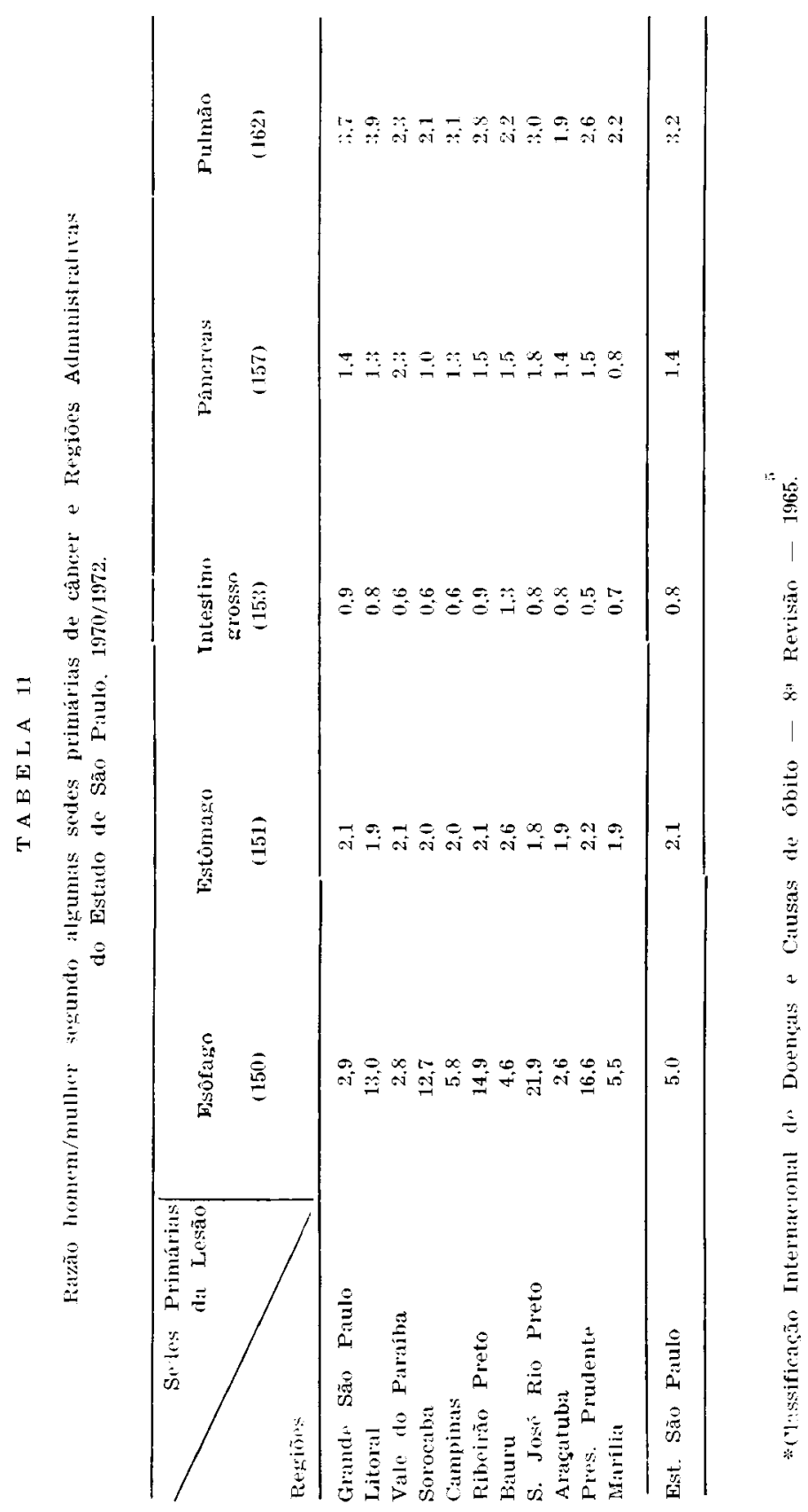


PAStorelo, E. F. \& GOTlieb, S. L. D. Mortalidade por câncer no Estado de São Paulo, Brasil - 1970/1972. Rev, Saúde públ., S. Paulo, 12:1-15, 1978.

tro vezes maior para o sexo masculino, ao passo que na Região de Araçatuba esse risco é somente duas vezes maior para os homens.

O tumor maligno do pâncreas apresentase com pequenas variações na razão homens/mulheres, com risco de morte em torno de 1,5 vezes maior para o sexo masculino.

Uma marcante diversificação surge no câncer do esôfago, notadamente um câncer masculino; us valores apresentados pela razão homens/mulheres variam entre 22 a 2,6 vezes, respectivamente nas Regiōes de São José do Rio Preto e Araçatuba.

Os padrōes aqui descritos relativos à comparação dos riscos de morrer entre homens e mulheres, para sedes primárias selecionadas de câncer, são os observados " chamando atenção aquele apresentado pelo câncer do esôfago que, em algumas Regiōes tem valores elevados, exigindo, talvez, estudos posteriores mais específicos.

\section{Considerações Finais}

Assim, vista a distribuição dos tumores malignos no Estado de São Paulo, em função de suas principais sedes primárias, pode-se estabelecer o possivel padrão de mortalidade por essa causa, para cada Região Administrativa. Todas apresentam, quer para o sexo masculino, quer para o sexo feminino, um padrão básico de mortalidade por câncer com predominância dos tumores do aparelho digestivo, notadamente câncer do estômago. Se bem que a etiologia permaneça ainda a ser demonstrada, estudos epidemiológicos têm apresentado no correr dos anos, inúmeros fatores associados ao câncer do estômago.* Nesse sentido, a associação deste particular tumor com fatores alimentares, como dietas ricas em carbohidratos e pobres em proteinas, frutas e vegetais, mostra-se consistente com as suas distribuições, tanto geográfica como sócio-econômica observadas. ${ }^{\ddagger}$ No sexo feminino, o câncer do útero como um todo aparece como importante componente do padrão básico de mortalidade por câncer para todas as Regiões Administrativas.
Apesar da análise global dos tumores malígnos do útero ter sido, neste estudo, efetuada em razão dos altos valores observados na categoria 182.9 (tumor maligno do útero sem especificar a parte), talvez, deva-se agora, calcados nas informaçoes e consideraçōes da Tabela 7 , admitir que um grande percentual dos tumores malígnos do útero são, possivelmente, tumores malignos do colo uterino. Tal como o câncer do estômago, este tumor apresenta a variável sócio-econômica como um dos principais componentes do seu quadro epidemiológico 3 . Neste sentido, havendo associação entre baixo nível sócio-econômico de uma população com o aumento de risco dos tumores do estômago e colo uterino, constata-se que, diante dos resultados aqui obtidos, o padrão básico de mortalidade por câncer nas Regiões Administrativas do Estado é compativel com a situação sócio-econômica ainda não elevada, em que se encontra o Estado de São Paulo, como um todo.

Por outro lado, en algumas Regiōes Administrativas do Estado, como a Grande São Paulo, Litoral, Campinas, Ribeirão Preto e Bauru, tumores malígnos da mama, pulnuão e próstata, típicos de situações sócio-econômicas mais elevadas ${ }^{1}$, já se constituem em importantes fatores de risco de morte. Esses fatos permitem assumir que algumas Regiões Administrativas, apesar de não fugirem ao padrão homogêneo, relativamente à mortalidade por câncer no Estado, apresentam a coexistência de tumores típicos de regiōes de nível sócio-econômico baixo e em elevaçāo, por constituirem-se em regiōes polo de desenvolvimento industrial do Estado (Figs. 1 e 2).

Neste ponto, cabe considerar que as diferenças aqui observadas quanto ao padrão de mortalidade por câncer nas Regiões Administrativas são reflexo da incidência desses tumores malígnos em função da exposição a agentes cancerígenos, bem como da estrutura populacional inerente à composição sócio-econômica demográfica de cada Região Administrativa. A duração da doença, variável dependente da qualidade 
PASTORELO, E. F. \& GOTLIEB, S. L. D. Mortalidade por câncer no Estado de São Paulo, Brasil - 1970/1972. Rev. Saúde públ., S. Panlo, 12:1-15, 1978.

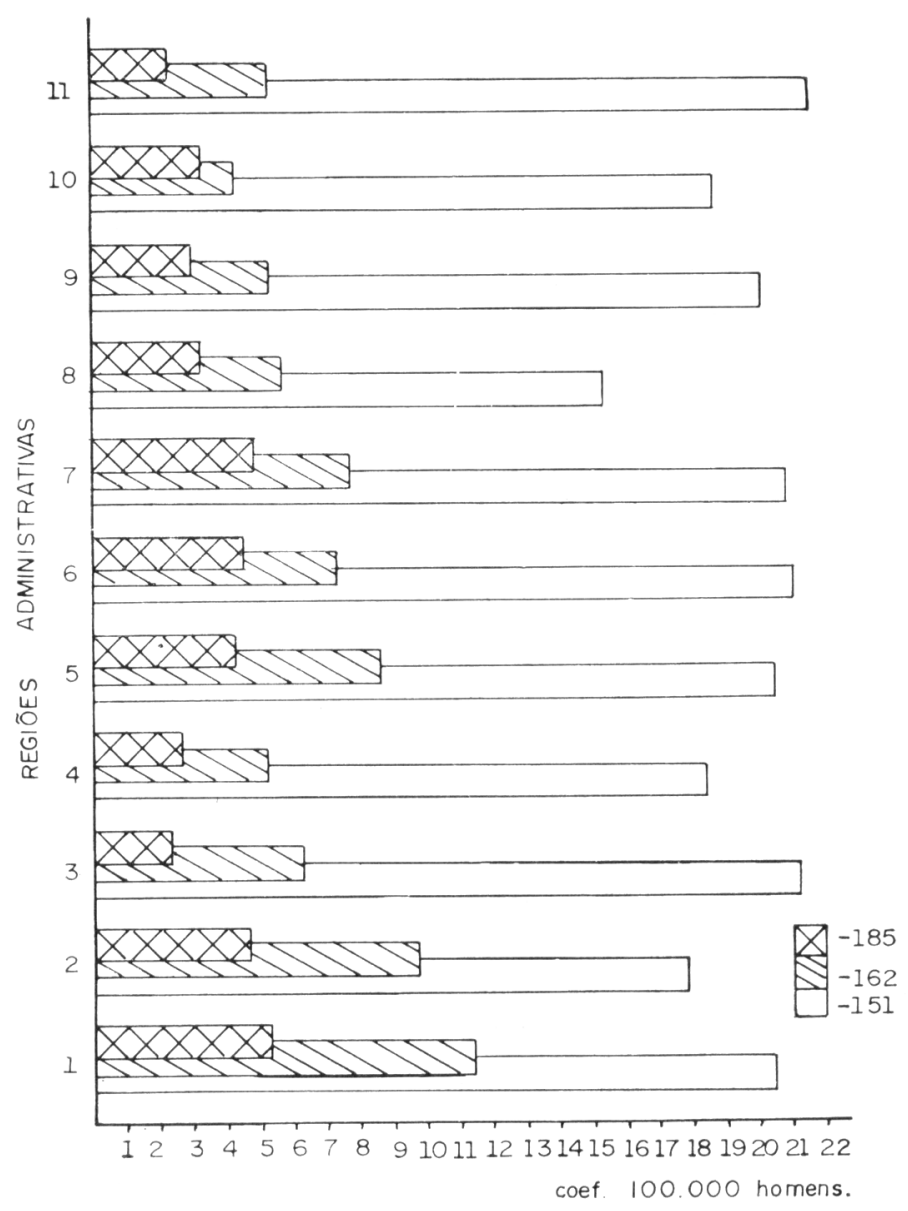

Fig. 1 - Coeficientes padronizados de mortalidade por câncer de estômago (151), câncer de pulmão (162) e câncer de próstata (185) no sexo masculino segundo as Regiōes Administrativas do Estado de São Paulo, 1970' 1972 (X 100.000 homens).

da atenção médica, também influi nestas estatisticas de mortalidade.

Assim, considerando-se a hierarquização das Regiões Administrativas do Estado de São Paulo, em relação a nível de Saúde, apresentada por Guedes 2 , constatou-se que as Regiões em transição aqui apontadas são as mesmas que se colocam nas primeiras posições da hierarquização supracitada. Tais Regiões apresentam as maiores notas nos indicadores de recursos de saúde, saneamento, fatores demográfico-educacionaleconômicos; indicadores estes compativeis com o aumento do risco de morte por tumores malígnos de localizações específicas de regiões sócio-econômicas mais desenvolvidas. 
PASTORELO, E. F. \& GOTLIEB. S. L. D. Mortalidade por câncer no Estado de São Paulo. Brasil - 1970/1972. Rev. Saúde publ., S. Paulo, 12:1-15, 1978.

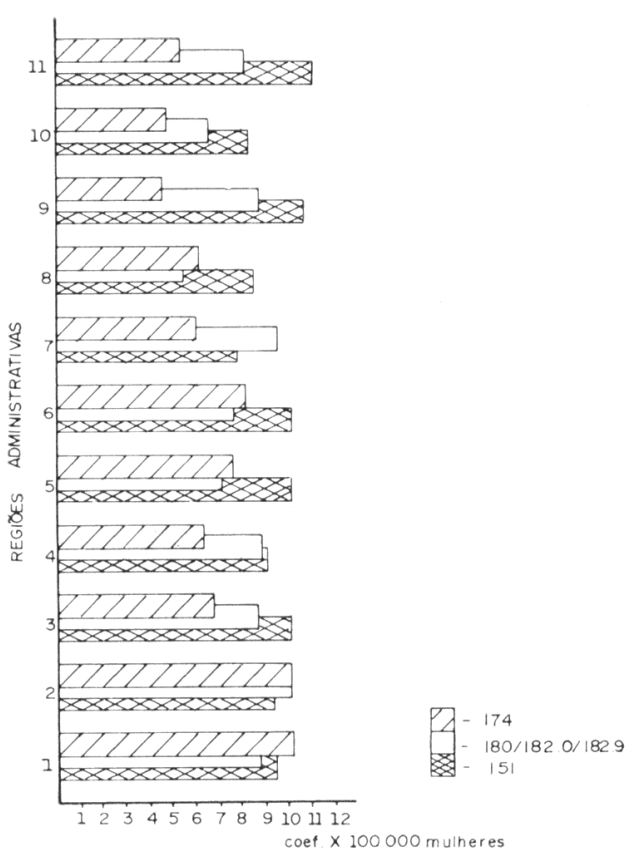

Fig. 2 - Coeficientes padronizados de mortalidade por câncer de estômago (151), câncer de útero como um todo (180, $182.0 \mathrm{e}$ 182.9) e câncer da mama (174) no sexo feminino segundo as Regiões Administrativas do Estado de Såo Paulo, 1970/1972 (X 100.000 mulheres).

\section{CONCLUSOES}

1. Os tumores malignos representaram a quinta e quarta causa de óbito, respectivamente para os homens e mulheres residentes no Estado de São Paulo em $1970 / 1972$.

2. Dentre os óbitos masculinos por câncer, a principal sede primária da lesão foi () estômago - $25 \%$ dos óbitos seguindo-se o pulmão $-11,4 \%$.

3. Dentre os óbitos femininos por câncer, a principal sede primária foi o estômago - $15,5 \%$ dos óbitos, seguindo-se mama - $14,1 \%$.
4. O coeficiente de mortalidade por câncer de estômago padronizado variou entre 15,40 e 21,56 por cem mil homens, respectivamente nas Regiões de São José do Rio Preto e Marília. O coeficiente de mortalidade por câncer do pulmão variou entre 4,35 e 11,41 por cem mil homens, respectivamente nas Regiões de Marilia e Grande São Paulo.

5. O coeficiente de mortalidade por câncer do estômago padronizado variou entre 7,91 e 11,11 por cem mil mulheres nas Regiōes de Bauru e Marilia, respectivamente, e o risco de morrer por câncer de mama variou entre 4,65 e 10,22 por cem mil mulheres, respectivamente, nas Regiōes de Araçatuba e Grande São Paulo. O útero como um todo foi, também, uma das principais sedes primárias da lesão, apresentando valores entre 5,55 e 10,09 por cem mil mulheres nas Regiões de São José do Rio Preto e Litoral.

6. Os resultados obtidos mostram um padrão básico de mortalidade por câncer, em relação às suas sedes primárias, nas Regiões Administrativas do Estado de São Paulo, compatível com situação sócio-econômica não elevada mas, em algumas dessas Regiōes (Grande São Paulo, Litoral, Campinas, Ribeirão Preto e Bauru) sedes primárias típicas de situação econômica mais elevada já se constituem em importantes fatores de risco de morte.

\section{AGRADECIMENTOS}

A Fernāo Dias de Lima, do Departamento) de Epidemiologia, pelos serviços de computação. 
PASTORELO, E. F. \& GOTLIEB, S. L. D. Mortalidade por câncer no Estado de São Paulo, Brasil - 1970/1972. Rev. Saũde públ., S. Paulo, 12:1-15, 1978.

RSPUB9/388

Pastorelo, E. F. \& Gotlieb, S. L. D. ICancer mortality in the State of S. Paulo, Brazil - 1970/1972.J Rev. Saúde púb1., S. Paulo, 12: $1-15,1978$.

ABSTRACT: The authors describe the distribution of cancer deaths according to the main anatomical sites of the primary tumor in the eleven Administrative Regions of the State of São Paulo, Brazil, during the period 1970/1972. Although having the same homogeneous pattern of cancer mortality of the State, some Regions show coexistence of tumours tipically of underdeveloped and developed areas. This could be explained by the fact that in such Regions there are some cities in a stage of high industrial development.

Uniterms: Neoplasms, S. Paulo, Brazil. Mortality.

\section{REFERENCIAS BIBLIOGRAFICAS}

1. CORREA, $P$. et al. The cancer registry of Cali, Colombia: epidemiologic studies of gastric cancer. In: GRUNDMANN, E. \& PEDERSEN, E., ed. Recent results in Cancer Research, New York, 1975. v. 50.

2. GUEDES, J. S. Contribuição para o estudo da evolução do nivel de saúde do Estado de São Paulo: analise das Regioes Administrativas (1950-1970). São Paulo, 1972. [Tese de Doutoramento - Faculdade de Saúde Pública da USP]

3. HILL, G. B. Cancer del utero: tendencia de la mortalidad desde 1950 . Cron. OMS, 30:201-6, 1976.

4. LOGAN, W. P. D. Cancers of the alimentary tract: international mortality trends. WHO Chron., 30:413-9, 1976.

5. ORGANIZAÇO PANAMERICANA DE SAƯDE. Manual de classificação estatistica internacional de doencas, lesōes e causas de bbito: 8a Revisão, 1965.
Washington, D. C., 1969. (Publ, cient., 190)

6. PASTORELO, E. F. \& GOTLIEB, S, L, D Estado de São Paulo, Brasil, 1970 a 1975 - Estimativas populacionais. Rev. Saúde públ., S. Paulo, 10(Supl. 1): $1-65,1976$.

7. PUFFER, R. R. \& GRIFFITH, G. W. Caracteristicas de la mortalidad urbana. Washington, D. C., Organización Panamericana de la Salud, 1968. (OPAS - Publ. cient., 151).

8. SCHOENBERG, B. S. \& BAILAR, J. C. Uterine malignancies of unespecified origin. Arch. environ. Hlth, 23:77-81. 1971.

9. SEGI, M. \& KURIHARA, M. Cancer mortality for selected sites in 24 countries (1966-6\%). Sendai, Japan Cancer Society, 1972.

Recebido para publicasāo em 02/06/1977. Aprovado para publicação em 14/07/1977. 